\title{
Tension Pneumomediastinum: A life-threatening condition in COVID-19 Patients
}

Nissar Shaikh $^{1}$, Gamal Al Ameri ${ }^{1}$, Muhsen Shaheen ${ }^{1}$, Wael Abdaljawad ${ }^{1}$, Sujith

Prabhakaran $^{1}$, Mohammad Alwraidat ${ }^{1}$, Ahmed soliman Mohamed ${ }^{1}$, Mohamad Khatib ${ }^{1}$, and Abdulqadir Nashwan ${ }^{1}$

${ }^{1}$ Hamad Medical Corporation

April 25, 2021

\begin{abstract}
Tension pneumomediastinum (TPM) is a rare but potentially fatal clinical entity. This leads to leakage of air into the mediastinal cavity and increased pressure on thoracic vessels, respiratory tract, and the heart. We report a series of five cases of COVID-19 complicating into acute respiratory distress syndrome and developing TPM.
\end{abstract}

Tension Pneumomediastinum: A life-threatening condition in COVID-19 Patients

Short title: Tension Pneumomediastinum \& COVID-19

Nissar Shaikh ${ }^{1}$, Gamal Al Ameri ${ }^{1}$, Muhsen Shaheen ${ }^{3}$, Wael I. Abdaljawad ${ }^{3}$, Sujith Prabhakaran $^{1}$, Mohammad Al Wraidat ${ }^{2}$, Ahmed S. Mohmed ${ }^{2}$, Mohamad Y. Khatib ${ }^{2}$, Abdulqadir J. Nashwan ${ }^{2}$

\section{Affiliations}

1. Surgical Intensive Care Department, Hamad General Hospital (HGH), Hamad Medical Corporation (HMC), Doha, Qatar

2. Critical Care Department, Hazm Mebaireek General Hospital (HMGH), Hamad Medical Corporation (HMC), Doha, Qatar

3. Medical Intensive Care Department, Hamad General Hospital (HGH), Hamad Medical Corporation (HMC), Doha, Qatar

Corresponding author:

Mr. Abdulqadir J. Nashwan

anashwan@hamad.qa

Tel: $(+974) 40240487$

Mob: (+974) 66473549

P.O.Box 3050 Doha, Qatar

\section{Abstract}

Tension pneumomediastinum (TPM) is a rare but potentially fatal clinical entity. This leads to leakage of air into the mediastinal cavity and increased pressure on thoracic vessels, respiratory tract, and the heart. 
We report a series of five cases of COVID-19 complicating into acute respiratory distress syndrome and developing TPM.

Keywords : Acute respiratory distress syndrome, COVID-19, chest drain, invasive ventilation, tension pneumomediastinum.

\section{Key Clinical Message :}

COVID-19 pneumonia patients with ARDS requiring invasive ventilation and prone positioning can complicate into TPM. Early recognition is vital in management as it may lead to serious complications as cardiac arrest.

\section{Background}

Pneumo-mediastinum is usually a benign condition defined as the presence of air in the mediastinum. However, in rare cases, excessive air in this space can increase pressure on vital cardiovascular and respiratory systems causing cardiovascular collapse. This phenomenon is known as tension pneumomediastinum (TPM). TPM is a potentially fatal clinical condition, initially described in the 1940s frequently in pulmonary tuberculosis patients. ${ }^{1}$ Severe COVID-19 pneumonia may progress to full-blown Acute respiratory distress syndrome within a short period of time. These patients require invasive ventilation with high ventilator settings to maintain their oxygenation requirements. Hence these patients are at a higher risk of developing pneumomediastinum and eventually tension pneumomediastinum (TPM). The literature search revealed one case report TPM and a case series titled "pneumomediastinum in COVID-19 patients". ${ }^{2,3}$ We report another case series describing five patients of COVID-19 pneumonia who developed tension pneumomediastinum while being given invasive ventilation.

\section{Case Series}

\section{Case 1}

A 51-year-old man without a history of previous medical illness presented in May 2020 with severe COVID19 pneumonia. He had shortness of breath and low oxygen saturation, requiring noninvasive ventilation in the initial period. His chest radiographs showed bilateral peripheral infiltrates. He was managed as per the hospital COVID-19 protocol at that time. Initially, he was advised self-prone position with oxygen supplementation; but on the 4th day, his condition deteriorated, requiring intubation and ventilation. During his stay in ICU, he developed a cytokine storm requiring IL-6 antagonist Tocilizumab and steroids. He had a prolonged course of invasive ventilation during which his position was changed to prone multiple times. He received convalescent plasma and was successfully extubated on the 15 th day after improvement in ventilatory settings. He remained stable for the next 4 days but developed severe sepsis, leading to septic shock and ARDS, eventually leading to reintubation. Sputum cultures grewStenotrophomonas maltophilia and Klebsiella, which was extended-spectrum beta-lactamase resistant (ESBL).

Ventilator support continued for another 8 days, and on day 28th after his admission, he developed extensive lower neck and chest wall subcutaneous emphysema. Chest x-ray (CXR) showed pneumo-mediastinum (Figure 1). No evident pneumothorax was noted on the CXR on that day. During this period, he had been on high ventilatory settings, including controlled mandatory ventilation (CMV) with Fio2 50\%, tidal volume $350 \mathrm{ml}, \mathrm{PEEP}$ of $8 \mathrm{mmHg}$, and Respiratory rate of 30/minute.

CT scan was done, which showed extensive pneumomediastinum and bilateral pneumothorax. A small suspicious area of possible disintegration and discontinuation within the right posterolateral wall of the lower part of the trachea was noticed (Tracheal tear). Bilateral diffuse ground-glass opacities were observed bilaterally in the lung fields, which were consistent with his underlying ARDS. (Figure 2)

Right side chest drain was inserted to relieve the tension pneumothorax. His condition deteriorated in the next few days due to multi-organ failure, and the patient expired on day 36 in the ICU.

Case 2 
A 71-year-old man with a past medical history of osteoarthritis and hypertension bring managed with amlodipine presented to the hospital with signs of COVID-19 pneumonia and moderate ARDS. He was initially managed with a non-rebreathing oxygen mask, self-proning, and noninvasive ventilation. He was managed with antibiotics, antivirals, steroids, and as he showed signs of cytokine storm, IL-6 antagonist Tocilizumab was given. Later, he also received convalescent plasma from recovered COVID-19 donors. The management was done according to the hospital's COVID-19 management guidelines. During his course in the hospital, he developed hospital-acquired secondary pneumonia fromEnterobacter cloacae, Candida, and Acinetobacter baumanni. Bronchoalveolar lavage grew Stenotrophomonas maltophilia. He was intubated and ventilated on day 32 in the hospital due to worsening of ARDS, sepsis, multiorgan dysfunction syndrome (MODS).

Ventilatory requirements remained high during this period and on day 37, his chest radiographs showed pneumomediastinum, subcutaneous emphysema with no pneumothorax. His ventilator settings on that day were CMV mode with FiO2 40\%, positive end-expiratory pressure

(PEEP) of $8 \mathrm{mmHg}$, tidal volume $370 \mathrm{ml}$, and respiratory rate of $26 /$ minute.

Computerized topography (CT) scan showed extensive surgical emphysema in the neck and anterior-lateral aspect of the chest wall, which was more prominent in the right side. Extensive tension pneumo-mediastinum was noted extending from the superior mediastinum, anterior mediastinum, posterior mediastinum and extending even to the retroperitoneum space surrounding the pancreas and left kidney. No evidence of pneumoperitoneum was found. Bilateral thin rim of pneumothorax was noted slightly more on the left side. No evidence of lung collapse was seen in the CT scan.

Conservative management was adopted for his pneumo-mediastinum by reducing the ventilator pressures and close observation. The condition resolved over time. He was tracheostomized on day 12 due to a prolonged ventilatory course. He developed recurrent sepsis during his stay in the ICU; however, due to timely management, his condition improved over time. The patient was discharged from the ICU on day 29.

Case 3

A 58-year-old man was diagnosed with COVID-19 pneumonia. He had a complicated past medical history, including diabetes mellitus, end-stage renal disease, status-post renal transplant, and pulmonary tuberculosis 3 years back. He had completed full course of anti-tuberculosis. He presented with worsening shortness of breath and severe pneumonia, requiring intubation on 3rd day of admission. During his stay in the ICU, he developed severe sepsis, MODS requiring antibiotics, antivirals, and steroids. He also showed signs of cytokine storm requiring IL-6 antagonist Tocilizumab. Later, he also received convalescent plasma. All the management was done according to the hospital's COVID-19 management protocol. Prone positioning was done several times during his ICU stay. His tracheal aspirate culture grew Aspergillus ochraceus and Candida tropicalis. On day 22, the CXR showed subcutaneous emphysema with no pneumothorax but was suspicious of pneumo-mediastinum. His ventilatory settings were CMV with Fio2 40\%, PEEP 10, Tidal volume 4-6 $\mathrm{ml} / \mathrm{kg}$, and a high respiratory rate. CT thorax revealed a significant amount of air within the mediastinum (tension pneumo-mediastinum). Anterior chest wall emphysema was also noted. There was no evidence of pleural effusion or pneumothorax. Bilateral diffuse ground-glass appearance and airspace opacities involving all lung lobes were seen. The pneumo-mediastinum was managed conservatively and was totally resolved without any intervention at that time. His condition deteriorated further during his stay in ICU due to severe sepsis and ARDS. Vasopressor requirements increased, and he suffered cardiac arrest on day 32. The cardiac arrest was reverted, but due to severe brain insults, his condition did not improve. He was transferred from the ICU for long-term care and rehabilitation.

Case 4

A 63-year-old man with a past medical history of type-2 diabetes, diabetic nephropathy presented with sudden deterioration of cognitive function, generalized fatigability, and hematuria. He was COVID-19 positive upon admission. His MRI showed infratentorial microhemorrhages. His condition deteriorated and he devel- 
oped COVID-19 pneumonia and ARDS requiring intubation on day 18 of admission. He was managed with antiviral, antibiotics, as he also showed signs of cytokine storm requiring IL-6 antagonist Tocilizumab and steroids. 10 days after intubation, his CXR showed signs of pneumoperitoneum and anterior extraperitoneal air. The ventilator settings included pressure control mode with Fio2 60\%, PEEP 8, pressure control 32 and respiratory rate of 28/min. His sputum culture was growing Klebsiella, Candida albicans, and Serratia marcescens .

CT scan showed evidence of mild lower neck surgical emphysema more to the right lateral side. Extensive tension pneumo-mediastinum was noted extending from the superior mediastinum, down anteriorly and posteriorly to the pre-crural and pre-cardiac space causing cardiac tension. This pneumo-mediastinum extended inferiorly to the anterior retroperitoneal space. No intraperitoneal free air was seen. Diffuse mosaic ground-glass attenuation of both lungs was noted which was consisted with ARDS.

Pneumomediastinum was managed conservatively and he recovered from this. Later he developed severe sepsis, multi-organ dysfunction and lung fibrosis and unfortunately expired one month later.

Case 5

A 51-year-old man with past medical history of type-2 diabetes had worsening COVID-19 pneumonia and ARSA requiring intubation on day 10. He developed sepsis and multi-organ dysfunction. He was managed with antiviral, antibiotics, Tocilizumab and steroids. Two days after intubation, his CXR showed left side subcutaneous emphysema and signs of pneumomediastinum. His ventilator settings were CMV mode with tidal volume 390, PEEP 8 and Fio2 of 50\%. During his course in ICU, he went into severe sepsis, MODS requiring antibiotics, antivirals, and steroids. He also showed signs of cytokine storm requiring IL-6 antagonist Tocilizumab.

CT showed extensive pneumo-mediastinum and subcutaneous emphysema with mild pneumo-thoraces bilaterally. The surgical emphysema extended into the neck. The pneumo-mediastinum extended into the abdomen and appeared anteriorly in the extraperitoneal region. Bilateral segmental branches of the main pulmonary arteries, going towards the lower lobes, showed filling defects.

As he also had significant pneumothorax, the right-sided chest tube was inserted. A few hours later, he had cardiac arrest due to refractory hypoxia requiring ECMO support. He responded to the management and was decannulated from ECMO after 29 days. His sputum grew Enterococcus fecalis , Klebsiella, and Serratia marcescens. He recovered after a prolonged course in ICU and was discharged for rehabilitation.

All five cases key lines are presented in table1.

\section{Discussion}

Tension pneumomediastinum (TPM) is caused by direct injury or barotrauma to the tracheobronchial tree, alveoli, or esophagus, leading to air leakage into the mediastinum and causing tension in the closed cavity. This may cause compression of large vessels, heart, and lungs leading to cardiovascular and respiratory compromise, which can be a threat to the patient's life. ${ }^{2,4}$ COVID-19 infection attacks initially the respiratory system causing pneumonia. Complications include acute respiratory distress syndrome requiring invasive ventilation with higher or maximum ventilator settings. This carries an increased risk of barotrauma to the lungs and tracheobronchial tree. There is not much literature about COVID-19 pneumonia and complicating into TPM or pneumomediastinum, there are only one case report of TPM, and a series of 8 cases of pneumomediastinum are described till date. ${ }^{2,3,5-7}$ COVID-19 infection is known to cause airway inflammation and edema, which puts these patients at a higher risk of airway tract injuries following instrumentation. ${ }^{3}$ Placement of a large size endotracheal tube (ETT) also carries a risk of development of TPM in COVID-19 patients. ${ }^{3}$ Although in our case series, only one patient had a tracheal injury but three patients required reintubation due to secondary bacterial and fungal pneumonia with increased risk for TPM.

All our patients were given intermittent prone positioning after the intubation as a therapeutic approach for COVID19 respiratory failure. Prone positioning in acute respiratory distress patients is known to be a risk 
factor for the development of pneumomediastinum and TPM. ${ }^{8}$ Wali et al. described in their case series that one of their COVID-19 patients developed pneumomediastinum immediately after prone positioning. ${ }^{3}$ All our patients had pneumo-mediastinum at day 10 post-intubation or later. They had secondary bacterial and or fungal pulmonary infections. The majority of our patients also had complex previous medical histories, which may be contributing to their frail condition.

For the diagnosis of TPM apart from hemodynamic instability, imaging studies are confirmatory. Initial CXR will show the presence of air in mediastinum, around or earth heart sign due to collapsed and restricted filling of the heart chambers. ${ }^{9}$ Sometimes, it's difficult to see the heart shadow in the x-ray of TPM patients; hence it is called "vanished heart sign". ${ }^{10}$ A computerized tomography of the chest will show more detailed extension of air in the mediastinum, including the retromediastinum. ${ }^{3}$

TPM is treated with insertion of suprasternal drains or through xiphisternum to decompress the mediastinum. The conservative management includes reducing airway pressures, allowing permissive hypercapnia and denitrogenation of the mediastinum air by increasing the percentage of oxygen supplementation. ${ }^{2,3}$ Two of our patients were managed by insertion of the intercostal drain and three patients were managed conservatively. One patient required extracorporeal membrane oxygenation (ECMO) therapy. One of conservatively managed and one patient managed with drainage of TPM died later due to other complications.

\section{Conclusion}

Concluding lines from our case series is that patients with COVID-19 acute respiratory distress syndrome with secondary infection can develop Tension pneumo-mediastinum. TPM can cause cardiac arrest. Management should be prompt in decompensated cases with the insertion of drains or conservatively in stable patients. ECMO can be used in TPM patients with ARDS who are not responding to invasive ventilation.

\section{Abbreviations}

ARDS: Acute respiratory distress syndrome

TPM: Tension pneumomediastinum

ESBL: Extended-spectrum beta-lactamase resistant

CMV: Controlled mandatory ventilation

MODS: Multiorgan dysfunction syndrome

PEEP: Positive end-expiratory pressure

ECMO: Extracorporeal membrane oxygenation

\section{Declarations}

\section{Ethics approval and consent to participate}

The article describes a case series. Therefore, no additional permission from our Ethics Committee was required (MRC-04-20-1191).

\section{Consent for publication}

The consents for publication were obtained from all included patients.

\section{Availability of data and material}

All data generated or analyzed during this study are included in this published article.

\section{Competing interests}

The authors declare that they have no competing interests.

\section{Funding}


This study was not funded.

\section{Authors' contributions}

Data Collection: GAA, MAW

Literature Search: NSH, MSH, WIA, SPR

Manuscript Preparation: NSH, AJN, ASM, MKH.

All authors read and approved the final manuscript.

\section{Acknowledgments}

Open Access funding provided by the Qatar National Library.

\section{References}

1. van Stiegmann G, Brantigan CO, Hopeman AR. Tension penumomediastinum. Arch Surg 1977; 112(10):1212-15

2. Campisi A, Poletti V, Ciarrocchi AP, et alTension pneumomediastinum in patients with COVID-19Thorax 2020;75:1130-1131

3. Wali A, Rizzo V, Bille A, Routledge T, Chambers A. Pneumomediastinum following intubation in COVID19 patients: a case series. Anaesthesia2020; 75: 1076-1081

4. Stella F, Rossi E, Tosato F. Subcutaneous emphysema and tension pneumomediastinum. Emerg Med J. 2013; 30(7):604

5. Zhou C, Gao C, Xie Y, Xu M. COVID-19 with spontaneous pneumomediastinum. Lancet Infectious Diseases 2020; 20: 510

6. Sun R, Liu H, Wang X. Mediastinal emphysema, giant bulla, and pneumothorax developed during the course of COVID-19 pneumonia. Korean Journal of Radiology 2020; 21: 541- 4

7. Wang J, Su X, Zhang T, Zheng C. Spontaneous pneumomediastinum: a probable unusual complication of coronavirus disease 2019 (COVID-19) pneumonia. Korean Journal of Radiology 2020; 21: 627- 8

8. Scholten EL, Beitler JR, Prisk GK, Malhotra A. Treatment of ARDS with prone positioning. Chest 2017; 151: $215-24$

9. Carillo A G O, Caballero GB, Carretero MAC. The Earth-Heart sign: a new diagnostic finding in a patient with tension pneumomediastinum. Lancet Case report2014; 383: P486

10. Hyun Jin Cho, Mea-Young Chang, Shin Kwang Kang, Jae Hyeon Yu , Myung Hoon Na, Min-Woong Kang. A Vanished Heart: Tension Pneumomediastinum. Am J Respir Crit Care Med2015; 192(9):1130

\section{Tables and Figures}

Table1: The clinical characteristics and outcomes of the 5 cases

Figure1: Chest x-ray

Figure2: Chest CT-scan

\section{Hosted file}

Fig1.pdf available at https://authorea.com/users/409875/articles/519420-tensionpneumomediastinum-a-life-threatening-condition-in-covid-19-patients

\section{Hosted file}


Fig2.pdf available at https://authorea.com/users/409875/articles/519420-tensionpneumomediastinum-a-life-threatening-condition-in-covid-19-patients 